\title{
Comparison of core decompression and porous tantalum rod implantation with conservative treatment for avascular necrosis of the femoral head: A minimum 18 month follow-up study
}

\author{
YUAN FANG $^{1}$, CHANGRONG DING $^{2}$, YINGZHEN WANG $^{1}$ and HAINING ZHANG ${ }^{1}$ \\ Departments of ${ }^{1}$ Joint Surgery and ${ }^{2}$ Electrocardiogram, \\ The Affiliated Hospital of Qingdao University, Qingdao, Shandong 266000, P.R. China
}

Received June 3, 2019; Accepted February 26, 2020

DOI: $10.3892 /$ etm.2020.8669

\begin{abstract}
Porous tantalum rod implantation is a novel surgical method that is used to treat avascular necrosis (AVN) of the femoral head (hip). In the present study, the results of core decompression and tantalum rod implantation were compared with non-surgical treatment for AVN, and the survivorship of the femoral head was evaluated. In total, 60 patients with AVN femoral head were recruited and analysed. Non-surgical treatment was selected by 30 patients (41 hips), 7 with a Ficat score of I and 23 with a score of II. Non-surgical treatment included celecoxib, salvia miltiorrhiza and tetramethylypyrazine and a reduction in weight-bearing activities. Surgical treatment and porous tantalum rod implantation were selected by 30 patients (41 hips), 10 with a Ficat score of I and 20 with a score of II. After follow-up (average: 33.5 months), patients were evaluated by assessing post-operative complications, radiology, hip survivorship and Harris hip score. In the surgical group, pre-operative symptoms were significantly alleviated. No complications, including infection, delayed healing or fractures were reported. Final follow-up rates of femoral head survivorship were $4.9 \%$ in the non-surgical group and $36.7 \%$ in the surgical group. The Harris hip score was significantly improved following surgery when compared with non-surgical treatment $(\mathrm{P}<0.05)$. The results indicated that core decompression and porous tantalum rod implantation are beneficial short- and mid-term treatment methods for AVN of the femoral head.
\end{abstract}

Correspondence to: Dr Haining Zhang, Department of Joint Surgery, The Affiliated Hospital of Qingdao University, 59 Haier Road, Qingdao, Shandong 266000, P.R. China

E-mail: zhhaining2019@163.com

Abbreviations: AVN, avascular necrosis; THA, total hip arthroplasty

Key words: femoral head necrosis, tantalum, survival rate

\section{Introduction}

The majority of patients with avascular necrosis (AVN) of the femoral head (hip) experience collapse of the subchondral bone, leading to osteoarthritis $(1,2)$. Due to this, the preservation of the femoral head in the early stages of osteonecrosis is a primary objective. However, to the best of our knowledge, a consensus regarding the best approach to this is lacking.

Surgical intervention is more effective when it is performed prior to the collapse of the femoral head, especially in patients $<48$ years old (3). For AVN, treatment strategies include subchondral bone drilling, core decompression, vascularized bone-grafting, femoral osteotomy, surface replacement and total hip arthroplasty (THA) (4). However, these treatment options have a number of limitations. Core decompression results in a deficiency of subchondral plate support for the weight load, leading to inconsistent outcomes (5). Vascularized fibular grafting is limited by the morbidity associated with graft harvest and additional time required for surgery and potential complications during rehabilitation (6). Pain, infection and a loose association with a prosthetic are limiting factors for hip arthroplasty (7).

A proposed alternative for AVN is a porous tantalum rod, which is a biomaterial used to support the subchondral bone of the femoral head, providing advantageous physical and mechanical properties. The modulus of elasticity of the implant is $3 \mathrm{GPa}$, which is between that of subchondral bone (1.5 GPa) and cortical bone (15 GPa) (8). Additionally, it is significantly less than that of a titanium alloy (110 GPa), which minimizes stress shielding (8). The surface of the rod has a high-volume porosity (75-80\%) with interconnected pores (mean; $430 \mu \mathrm{m}$ ) similar to bone, allowing for primary stability and rapid bone in-growth (9). Soft tissue has been indicated to grow on the rod for 4 weeks post-implantation in an animal model (10). Additionally, a biomechanical fatigue test demonstrated that the rod can bear 4-fold the bodyweights of humans and has an intensity that is 9.3 -fold greater than the pressure it resists post-implantation, permitting physiological load-bearing $(11,12)$. A previous study has suggested that beneficial results can be achieved in patients with an integral head outline (13). 
Therefore, the purpose of the present study was to evaluate the clinical results and survival rates of patients fitted with the porous tantalum rod system, which was used to treat early-stage osteonecrosis of the femoral head.

\section{Materials and methods}

Demographics. The present study was approved by the Ethics Committee of the Institutional Review Board of the Affiliated Hospital of Qingdao University. Written informed consent was obtained from all patients whose specimens and clinical information were used for the present study.

The inclusion criteria were as follows: i) Diagnosis of early non-traumatic AVN of the femoral head; and ii) the ability to withstand surgery. The exclusion criteria were as follows: i) A history of metal allergy; ii) an infection in the hip or another part of the body; and/or iii) previous hip surgery.

A total of 60 patients (82 hips) who had received treatment between June 2010 and March 2015 in the Affiliated Hospital of Qingdao University were retrospectively selected for analysis. Patients included in the study had AVN of the femoral head with normal morphology and joint space and were classified as Ficat stage I or II $(14,15)$. The patients' reported symptoms included hip pain, limp, limited range of motion and hip dysfunction. Changes in the density of the subchondral bone was discernible by radiographic evaluation and the configuration of the femoral heads were intact. None of the patients had undergone any prior treatment for osteonecrosis. The risk factors for AVN included corticosteroid application, excessive alcohol consumption and trauma.

X-ray and MRI scans of the hip joint were used to diagnose and stage AVN of the femoral head according to the Ficat classification. Considering economic cost, all patients were evaluated by x-ray during the follow-up.

The patients were classified into two groups based on the treatment they requested: The non-surgical treatment group and the surgical treatment group. The non-surgical treatment group included 30 patients (41 hips; 26 males and 4 females) with a mean age of 48.1 years (31-61 years). A total of 7 patients exhibited Ficat stage I and 23 exhibited stage II. A total of 19 cases ( 8 left side, 11 right side) exhibited ipsilateral disease and 11 patients exhibited bilateral disease. The surgical group included 30 patients (41 hips; 26 males and 4 females) with a mean age of 44.2 years (23-68 years). Of these patients, 10 were classified as Ficat stage I and 20 as stage II. A total of 21 patients received a porous tantalum implant on one side (9 left side, 12 right side) and 9 received the implant bilaterally.

Treatment. In the non-surgical treatment group, celecoxib, salvia miltiorrhiza and tetramethylypyrazine were applied. The total course of the treatment was 6 weeks. Celecoxib (Pfizer, Inc.) was orally administered at a dose of $200 \mathrm{mg} /$ day for 6 weeks for analgesia during the entire course. Additionally, $10 \mathrm{ml}$ Salvia miltiorrhiza (Sanhome Pharmaceutical Co, Ltd.) and $20 \mathrm{mg}$ tetramethylypyrazine (Zhengzhou Cheuk-Fung pharmaceutical Co., Ltd.) diluted with $500 \mathrm{ml}$ of $10 \%$ glucose was concurrently administered intravenously daily for 10 days. After another 2 weeks of celecoxib treatment only, the regimen of Salvia miltiorrhiza and $20 \mathrm{mg}$ tetramethylypyrazine injection was repeated for an additional 10 days. Patients were advised to walk with two crutches to avoid full weight-bearing for at least three months and to walk $<1,000 \mathrm{~m} /$ day. Furthermore, patients were asked to reduce their daily activities to limit hip movement.

In the surgical treatment group, necrotic bone was located using x-ray prior to surgery. Epidural anaesthesia was administered to 20 patients with ropivacaine (Humanwell Healthcare Co., Ltd.) while the remaining 10 underwent endotracheal anaesthesia with sevoflurane (Lunan Pharmaceutical Group, Co., Ltd.). Patients were placed in a supine position, where the affected hip joint was disinfected with tincture of iodine followed by deiodination with alcohol. A $4 \mathrm{~cm}$ longitudinal mid-lateral incision was made from the tip of the greater trochanter towards the femoral shaft. A $3.22 \mathrm{~mm}$ guidewire was drilled into the centre of the osteonecrotic lesion to the level of subchondral bone using fluoroscopic guidance, under which core compression was conducted using cannulated reamers to enlarge the diameter of the tunnel to a diameter of $10 \mathrm{~mm}$. The osteonecrotic bone was removed with a curette and the tunnel was irrigated. Following this, the length of the guidewire was measured and the tunnel was tapped. A porous tantalum rod (Trabecular Metal; Zimmer Biomet) with a diameter of $10 \mathrm{~mm}$ and a length of 70-140 mm was then inserted into the tunnel reaching the subchondral bone with the lateral portion of the rod abutting the lateral femoral cortex. The incision was closed in layers and no drainage tube was inserted.

Rehabilitation protocol began the day following surgery. To avoid weight-bearing, patients were allowed to walk with crutches for $48 \mathrm{~h}$ post-surgery. Hips could be flexed to $\sim 90^{\circ}$ after three weeks. Patients were permitted to start physiotherapy protocol and progress to full weight-bearing after fluoroscopic confirmation of the integration of the implant and host bone.

Functional assessment. Patients received regular follow-up at the clinic. Harris score (16), Ficat classification (15), symptoms, range of motion, activity and radiography were performed/determined pre-operatively and during the follow-up period. Additionally, hip survivorship, implant status and any complications were also recorded during the follow-up period. Follow-up was performed once every three months for the first year and twice per year following that. In cases where the follow-up time of the patients were $<24$ months, for example 18 months, then Harris scores would be recorded at 6 and 12 months up until the final follow-up time. Harris scores would not be recorded at 24 and 36 months in which case, as these patients are considered lost to follow up. Failure of the implant and hips in the non-surgical treatment group served as an indication for THA. Indications for THA included sustained pain and dysfunction of the hips or dissatisfaction with either the surgical or non-surgical treatment.

Statistical analysis. Statistical analysis of the data was performed using SPSS 17.0 software (SPSS, Inc.). All continuous variables were expressed as mean \pm standard deviation (SD). An unpaired t-test was used for the comparison of two groups. The Kaplan-Meier method was used to analyse hip survivorship. The difference between groups was determined using the log-rank test. $\mathrm{P}<0.05$ was considered to indicate a statistically significant difference. 
Table I. Comparison of Harris scores before and after treatments ${ }^{\mathrm{a}}$.

\begin{tabular}{lcccccc}
\hline Group & Prior to surgery & 6 months & 12 months & 24 months & 36 months & Final follow-up $^{\mathrm{b}}$ \\
\hline Non-surgical group & $60.50 \pm 5.20$ & $69.45 \pm 13.92$ & $67.87 \pm 14.42$ & $64.34 \pm 11.29$ & $60.72 \pm 11.93$ & $57.50 \pm 10.25$ \\
Surgery & $61.20 \pm 5.00$ & $86.06 \pm 11.59$ & $83.83 \pm 10.61$ & $78.95 \pm 12.34$ & $72.71 \pm 13.15$ & $68.63 \pm 17.11$ \\
P-value & $>0.05$ & $<0.05$ & $<0.05$ & $<0.05$ & $<0.05$ & $<0.05$ \\
\hline
\end{tabular}

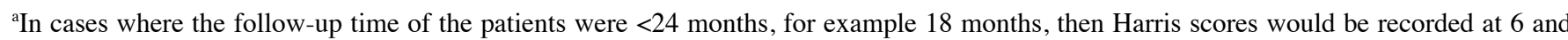
12 months up until the final follow-up time. Harris scores would not be recorded at 24 and 36 months in which case, as these patients are considered lost to follow up. ${ }^{\text {b}}$ Time range of final follow-up, 18-83 months.

\section{Results}

In the non-surgical treatment group, 27 cases (37 hips) were followed up and 3 cases ( 4 hips) were lost to follow-up. In the surgery group, 26 cases (36 hips) were followed up and 4 cases (5 hips) were lost to follow-up. The mean follow-up time was 33.5 months (range: 18-83 months). No difference in age was noted between groups $(\mathrm{P}>0.05)$. The average surgical time for tantalum rod implantation was $53 \mathrm{~min}$ (range; 40-80 $\mathrm{min}$ ). The mean blood loss was $80 \mathrm{ml}$ (range; $50-150 \mathrm{ml}$ ) and no infusion was required. No intra-operative or immediate post-operative complications occurred. The mean total hospitalization time, including those recorded before and after surgery, was 5 days (range; 3-7 days). No complications, such as infection of the incision, delayed healing, fracture or trochanteric bursitis, were recorded.

No difference in Harris score was identified between the non-surgical treatment and tantalum rod implant groups before treatment $(\mathrm{P}>0.05)$. Harris score increased at six months in the surgical group and then gradually decreased until the final follow-up (Table I). Significant differences in Harris score existed between the two groups at each stage of the follow-up $(\mathrm{P}<0.05)$.

Radiographic results at 12 months post-treatment revealed that 21 hips in the non-surgical treatment group exhibited an intact femoral head outline and a normal joint gap (Fig. 1A). The head appeared to have collapsed in the remaining 20 hips. In the surgical treatment group, the position of the tantalum rod in 31 hips was unchanged 12 months post-operatively, with a normal femoral head outline (Fig. 1B). However, partial collapse of the head was noted in the remaining 10 hips. At the final follow-up, only 5 hips in the non-surgical treatment group retained a normal femoral head outline and collapse of the head and narrowness of the joint gap were noted in the remaining 36 patients. THA was performed in 25 patients (28 hips) with Ficat stage IV due to accelerated pain, collapse of the femoral head and enlargement of the cyst region. In the surgical treatment group, 15 hips continued to exhibit normal implantation and a normal femoral head (Fig. 2A). The femoral head collapsed in the remaining 26 patients (Fig. 2B), including 4 patients (5 hips) who later underwent THA (Fig. 2C).

The significant differences between the two groups were verified using the log-rank test $(\log$ rank=20.330; $\mathrm{P}<0.001)$. The survival curve revealed that the survival rate of the patients in the non-surgical treatment group and surgical treatment group was 70.7 and $92.7 \%$ in the first year, respectively and 48.8 and $75.6 \%$ after 3 years, respectively. Final follow-up demonstrated a survival rate of $4.9 \%$ in the non-surgical treatment group and $36.7 \%$ in the surgical treatment group (Fig. 3).

The mean delay from the first treatment to THA was $17.64 \pm 5.82$ months (SD, months) in the non-surgical treatment group and $42.38 \pm 9.35$ months in the tantalum implantation group (Table II).

\section{Discussion}

In the present study, the follow-up data of patients with early AVN of the femoral head who received either non-surgical treatment or tantalum rod implantation was retrospectively analysed. The results indicated that porous tantalum rod implantation improved hip joint function to a certain extent, effectively delaying the requirement for THA caused by collapse of the femoral head and the narrowing of the joint gap and did not increase the risk of associated surgical complications.

Indications for THA treatment include collapse of the femoral head and narrowing of the joint space, demonstrating the importance of the outline of the femoral head (17). Therefore, the outline of the femoral head and hip joint must be preserved prior to collapse of the femoral head and subchondral plate, especially in patients $<48$ years old (18). Previous research has indicated that a subchondral bone buttress may be effective in halting or preventing the progression of osteonecrosis of the femoral head (19). Due to their minimally invasive characteristics, subchondral bone drilling and decompression were popular means of treatment despite their potential to weaken the cancellous bone within and adjacent to the necrotic area (20).

Mechanical support of the femoral head is necessary for weight-bearing and to delay disease progression, especially in patients with early-stage disease (21). This understanding has led to the development of bone transplant with or without blood supply and free fibular transplantation to prevent femoral head collapse (22). However, inconsistent effects and clinical results, shortage of mechanical support and aggressive secondary trauma has limited the achievement of satisfactory results (23).

An advantage of the porous tantalum rod is that the implant is minimally invasive and avoids the morbidity associated with vascularized and non-vascularized bone-grafting strategies, such as extensive surgical procedures, protected weight-bearing or risk of disease transmission (24). Veillette et al (25) evaluated the results of the tantalum rod in 44 patients (60 hips; mean age; 35 years) with a mean 

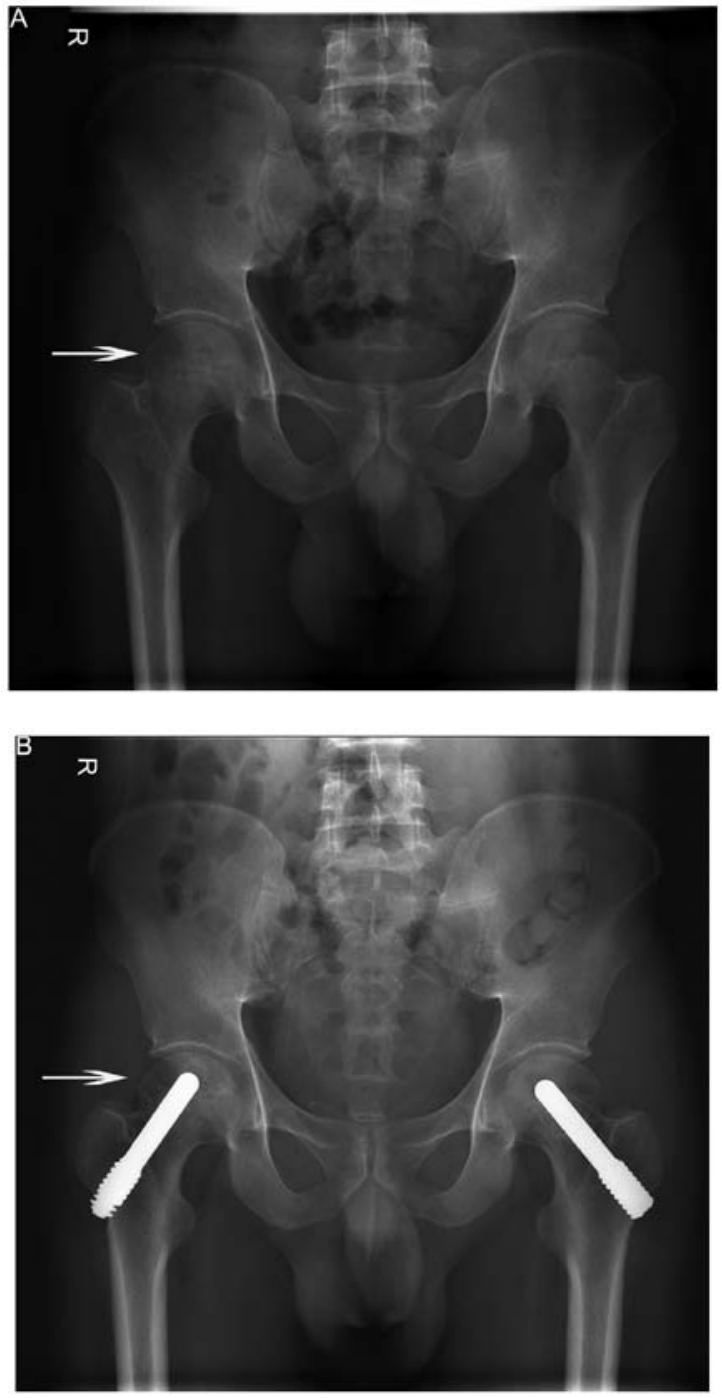

Figure 1. A 44-year old male patient exhibiting bilateral Ficat stage II disease. (A) Pre-therapy. (B) Post-operatively (12 months) after the implantation of porous tantalum rod. An x-ray revealed that the position of the tantalum rod was unchanged and that the femoral head outline was normal. $\mathrm{R}$ indicates the right side.

follow-up period of 24 months. According to the Steinberg classification (26), 1 hip (2\%) was classified as stage I, 49 hips (84\%) were classified as stage II and 8 (14\%) hips were classified as stage III. The overall survivorship rates were $91.8 \%$ at 12 months, $81.7 \%$ at 24 months and $68.1 \%$ at 48 months. A total of 9 hips (15.5\%) were converted to THA. Shuler et al (27) compared the results between vascularized fibular grafts and tantalum rod implants. No patient required patient-controlled analgesia or transfusion. In the tantalum implant group, the mean blood loss and operative time were reduced compared with those in the fibular graft group. All surviving implants (86\%) resulted in good to excellent outcomes. Kaplan-Meier analysis at 39 months revealed 86 and 67\% survivorship rates for implant and fibular grafts, respectively. A modified technique, including bone marrow mesenchymal stem cells and vascularized iliac grafting with a tantalum rod, was used in 24 patients with end-stage necrosis of the femoral head. After 64.35 months of follow-up, only five hips were converted to THA. The
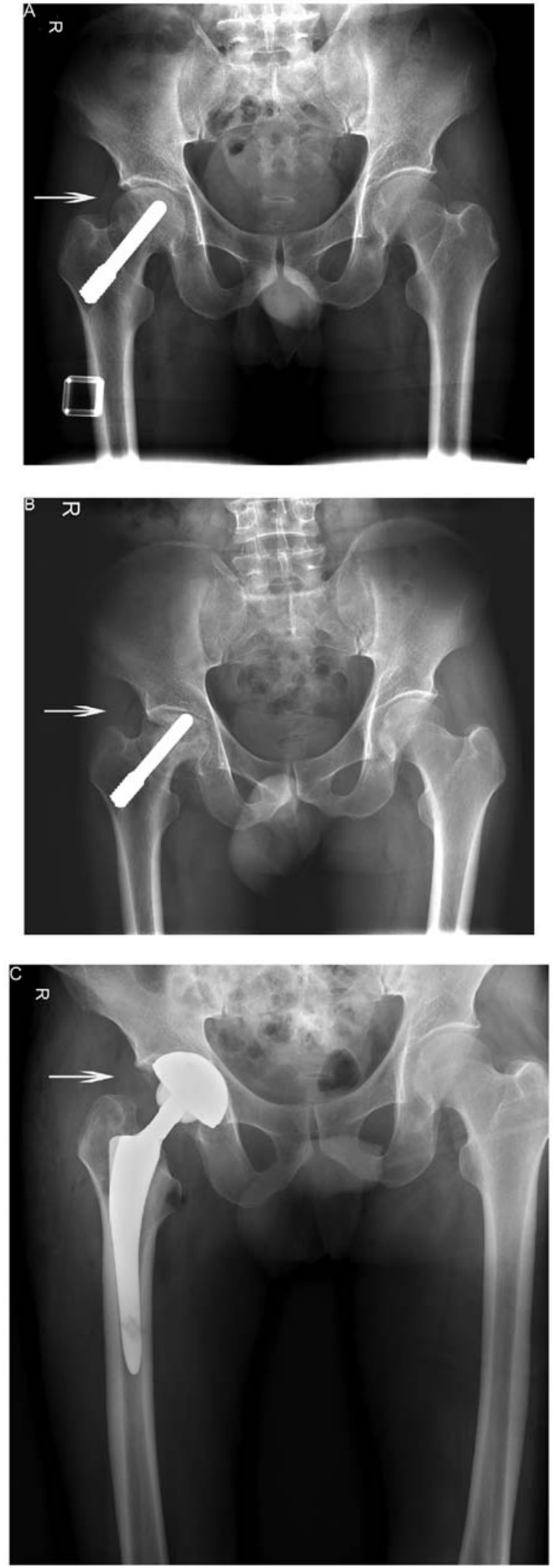

Figure 2. A 52-year-old female patient exhibiting right side Ficat stage II disease. (A) Post-operatively (12 months) after the implantation of a porous tantalum rod. An x-ray revealed that the position of the tantalum rod was unchanged and the femoral head outline was normal. (B) Post-operatively (15 months) after the implantation of porous tantalum rod. An x-ray revealed that the position of the tantalum rod was unchanged, whereas the femoral head on the right side had collapsed. (C) A total of 12 months after total hip arthroplasty. An x-ray revealed that the prosthesis was not loose and in a good position. $\mathrm{R}$ indicates the right side. 
Table II. Mean delay from the first treatment to THA.

\begin{tabular}{lccc}
\hline Group & $\begin{array}{c}\text { Non-surgical } \\
\text { group }\end{array}$ & Surgical group & P-value \\
\hline Time (months) & $17.64 \pm 5.85$ & $42.38 \pm 9.35$ & $<0.001$ \\
\hline
\end{tabular}

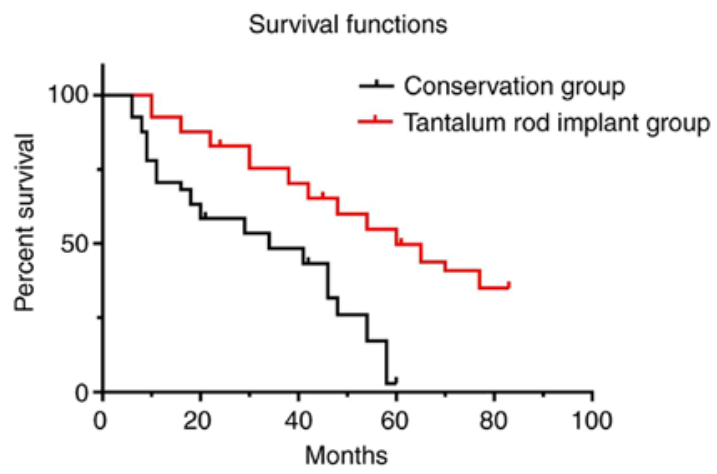

Figure 3. Survivorship curves. The survival rates of the patients in the non-surgical treatment and surgical treatment groups were found to be 70.7 and $92.7 \%$ in the first year, respectively; 48.8 and $75.6 \%$ after 3 years, respectively. The final follow-up survival rate was $4.9 \%$ in the non-surgical treatment group and $36.7 \%$ in the surgical treatment group.

success rate of this method was $89.47 \%$ for the Association Research Circulation Osseous (ARCO) stage III and $75 \%$ for ARCO stage IV (28). A further study used a 3D finite element method to compare the tantalum rod with fibula implantation and the core decompression model (29). When the tantalum rod was inside the osteonecrotic area, shortening the implant or fibula implant reduced the collapse values of the femoral head when the necrotic area was large $\left(120^{\circ}\right)$. A meta-analysis of clinical trials for porous tantalum rod implantation, including six randomized controlled trials and controlled clinical trials with 256 patients, was performed to analyse the efficacy and safety of the procedure compared with vascularized or non-vascularized bone grafting (30). The results revealed that this less invasive method was more effective and tolerable in early-stage patients with a necrotic femoral head, with the advantages of a better Harris score, a significantly reduced incidence of femoral head collapse and reduced blood loss (30). These data demonstrated that a tantalum rod can provide structural support for the subchondral bone, in addition to core decompression and bone growth, due to the porous nature of the tantalum.

In the present study, the terminal time point for the final follow-up was established as collapse of the femoral head, indicating failure of the mechanical support by the tantalum implants and subchondral bone (31). THA was not selected as the criterion because certain patients would not accept the surgery, despite the presence of symptoms including pain and hip dysfunction, due to the fear of surgery or financial reasons. Due to this, the actual clinical results of the tantalum rod implant could have been overestimated if THA was selected as the failure criterion for survivorship analysis. In fact, the mechanical role of the tantalum rod in supporting the head was not applicable when collapse occurred.
Core decompression may be helpful to alleviate symptoms after surgery (32). A meta-analysis was employed to identify 22 studies using a single surgical core decompression technique (33). A similar procedure identified 8 studies that treated patients non-surgically (34). The success rates for core decompression were 84,63 and $29 \%$ for Steinberg stages I, II and III, respectively. Non-surgically treated patients with stage 0 , I, II and III (AVN) demonstrated success rates of 86, 61, 59 and $25 \%$, respectively. $\chi^{2}$-analysis revealed that the success rate of core decompression was significantly increased compared with that of non-surgical treatment for stage I hips only. A prospective study compared core decompression with tantalum rod insertion in 60 patients. No favourable results were observed for the tantalum rod compared with multiple small drill holes (35). An additional study assessed survivorship and prognostic factors for radiographic progression and conversion to THA after tantalum rod implantation (36). After a mean follow-up period of 44.8 months, $29.82 \%$ of the hips exhibited radiographic progression and $19.30 \%$ were converted to THA. The overall survivorship rate was $72.49 \%$ at 60 months post-operatively. Corticosteroid use and bone marrow oedema were predictors of radiographic progression and bone marrow oedema was an independent prognostic factor for conversion to THA (36). Tantalum rods have also been used in combination with intra-arterial infusion of peripheral blood stem cells mobilized by granulocyte-colony stimulating factor via the medial circumflex femoral artery (37). The results revealed that targeted intra-arterial infusion of stem cells could enhance the efficacy of the tantalum rod.

In the present study, patient symptoms were completely or partially alleviated after tantalum rod implantation, indicating a benefit of core decompression prior to rod implantation. Positive early clinical results were associated with the survivorship of femoral head, reduced complications, sufficient strength to allow physiological load-bearing capabilities and short hospital stay. Transparent line, loosening, translocation or abnormal bone density were not noted around the implant during the follow-up, demonstrating the primary and secondary stability of the rods. These results may be attributed to the notion that the modulus of elasticity in the rod was similar to that of donor bone and the distal threads of the rods and high frictional stability of the implant prevent loosening. In addition, all the patients included in the current study were classified as Ficat stage I or II, indicating that the shape of the femoral head was relatively normal without collapse. Collapse was not considered to be a rational indication for tantalum rod implantation, given that the rod itself does not provide the characteristics necessary for repair or reconstruction.

The non-surgical treatments used in the present study included medication and reduction in weight bearing. However, it was difficult for certain patients to strictly follow the strategy due to their reduced quality of life and daily activities, especially walking with double crutches. Salvia miltiorrhiza is thought to increase peripheral vascular flow, improve microcirculation, inhibit thrombosis and enhance soft tissue repair (38). This agent has been used in TCM to treat hypertension, cervical spondylosis and AVN of the femoral head (39). Similar pharmacological activity has been reported for tetramethylpyrazine, an ingredient derived from Ligusticum wallichii (40). This agent acts as a calcium 
channel blocker and has been used in TCM to treat cerebral embolism, insufficient cerebral blood supply and AVN of the femoral head with positive results $(41,42)$. However, the protective mechanism of this drug in osteonecrosis remains unclear. Survivorship of the affected femoral head in the non-surgical treatment group of the current study was lower compared with that in the surgical treatment group, indicating the poor results of non-surgical treatment and the protective advantage of subchondral support. However, the short- or mid-term protection for this implant and the difficulties of prosthesis re-implantation requires further analysis.

It was demonstrated in the present study that a porous tantalum rod could, to some extent, preserve the structural integrity of the femoral head and protect the subchondral bone plate from collapse. However, there are a number of limitations to tantalum rod implantation. Porous tantalum rod implantation is suitable for patients with a small femoral head necrosis area. Considering that a tantalum rod can only serve a point supporting role (diameter; $10 \mathrm{~mm}$ ), it is not suitable for patients with multiple foci and a large necrosis area (43). Another disadvantage of the tantalum rod is that it is expensive and as a foreign body implant, it may require removal when deep tissue infection occurs which will increase the risk of fracture (35). After the failure of tantalum rod implantation, it was converted to THA. Extracting a rod is technically demanding due to strong osseointegration of the porous tantalum rod (10). Challenges inclu0de increased blood loss, longer operative time, bone loss along the trajectory of the rod and the subsequent potential increased risk of femoral fracture $(44,45)$. For this reason, additional data and further prospective studies are required to support the conclusion that tantalum rod implantation is more effective than bone transplant or core decompression and to assess the risks, benefits and financial issues for patients.

In conclusion, when compared with non-surgical treatment, core decompression and porous tantalum rod implantation are suitable treatment methods for AVN of the femoral head with beneficial short- and mid-term results.

\section{Acknowledgements}

Not applicable.

\section{Funding}

The current study was supported by the National Natural Science Foundation of China (grant no. 81672197).

\section{Availability of data and materials}

The data sets used and/or analysed during the current study are available from the corresponding author on reasonable request.

\section{Authors' contributions}

$\mathrm{HZ}$ and YW designed the study. YF, CD and YW participated in the acquisition of samples and clinical data. CD and YF performed statistical analysis. YF drafted the manuscript. All authors read and approved the final manuscript.

\section{Ethics approval and consent to participate}

The present study was approved by the Ethics Committee of the Institutional Review Board of the Affiliated Hospital of Qingdao University (approval no. QYFYKYLL 2010-09; Qingdao, China). Written informed consent was obtained from all patients whose specimens and clinical information were used for the current study.

\section{Patient consent for publication}

Not applicable.

\section{Competing interests}

The authors declare that they have no competing interests.

\section{References}

1. Babis GC, Sakellariou V, Parvizi J and Soucacos P: Osteonecrosis of the femoral head. Orthopedics 34: 39, 2011.

2. Mont MA, Jones LC and Hungerford DS: Nontraumatic osteonecrosis of the femoral head: Ten years later. J Bone Joint Surg Am 88: 1117-1132, 2006.

3. Amanatullah DF, Strauss EJ and Di Cesare PE: Current management options for osteonecrosis of the femoral head: Part II, operative management. Am J Orthop (Belle Mead NJ) 40: E216-E225, 2011.

4. Rajpura A, Wright AC and Board TN: Medical management of osteonecrosis of the hip: A review. Hip Int 21: 385-392, 2011.

5. Malizos KN, Karantanas AH, Varitimidis SE, Dailiana ZH, Bargiotas K and Maris T: Osteonecrosis of the femoral head: Etiology, imaging and treatment. Eur J Radiol 63: 16-28, 2007.

6. Tripathy SK, Goyal T and Sen RK: Management of femoral head osteonecrosis: Current concepts. Indian J Orthop 49: 28-45, 2015.

7. Jones CA and Pohar S: Health-Related quality of life after total joint arthroplasty: A scoping review. Clin Geriatr Med 28: 395-429, 2012.

8. Lee GW, Park KS, Kim DY, Lee YM, Eshnazarov KE and Yoon TR: Results of total hip arthroplasty after core decompression with tantalum rod for osteonecrosis of the femoral head. Clin Orthop Surg 8: 38-44, 2016.

9. Zhang X, Wang J, Xiao J and Shi Z: Early failures of porous tantalum osteonecrosis implants: A case series with retrieval analysis. Int Orthop 40: 1827-1834, 2016.

10. Fernandez-Fairen M, Murcia A, Iglesias R, Sevilla P, Manero JM and Gil FJ: Analysis of tantalum implants used for avascular necrosis of the femoral head: A review of five retrieved specimens. J Appl Biomater Funct Mater 10: 29-36, 2012.

11. Floerkemeier T, Thorey F, Daentzer D, Lerch M, Klages P, Windhagen $\mathrm{H}$ and von Lewinski G: Clinical and radiological outcome of the treatment of osteonecrosis of the femoral head using the osteonecrosis intervention implant. Int Orthop 35: 489-495, 2011.

12. Liu G, Wang J, Yang S, Xu W, Ye S and Xia T: Effect of a porous tantalum rod on early and intermediate stages of necrosis of the femoral head. Biomed Mater 5: 065003, 2010.

13. Nadeau M, Seguin C, Theodoropoulos JS and Harvey EJ: Short term clinical outcome of a porous tantalum implant for the treatment of advanced osteonecrosis of the femoral head. Mcgill J Med 10: 4-10, 2007.

14. Schmitt-Sody M, Kirchhoff C, Mayer W, Goebel M and Jansson V: Avascular necrosis of the femoral head: Inter- and intraobserver variations of ficat and ARCO classifications. Int Orthop 32: 283-287, 2008.

15. Jawad MU, Haleem AA and Scully SP: In brief: Ficat classification: Avascular necrosis of the femoral head. Clin Orthop Relat Res 470: 2636-2639, 2012.

16. Ovre S, Sandvik L, Madsen JE and Roise O: Comparison of distribution, agreement and correlation between the original and modified merle d'Aubigne-Postel score and the harris hip score after acetabular fracture treatment: Moderate agreement, high ceiling effect and excellent correlation in 450 patients. Acta Orthop 76: 796-802, 2005. 
17. Lieberman JR, Engstrom SM, Meneghini RM and SooHoo NF: Which factors influence preservation of the osteonecrotic femoral head? Clin Orthop Relat Res 470: 525-534, 2012.

18. Zalavras CG and Lieberman JR: Osteonecrosis of the femoral head: Evaluation and treatment. J Am Acad Orthop Surg 22: 455-464, 2014

19. Microsurgery Department of the Orthopedics Branch of the Chinese Medical Doctor Association, Group from the Osteonecrosis, Bone Defect Branch of the Chinese Association of Reparative, Reconstructive Surgery, Microsurgery and Reconstructive Surgery Group of the Orthopedics Branch of the Chinese Medical Association: Chinese guideline for the diagnosis and treatment of osteonecrosis of the femoral head in adults. Orthop Surg 9: 3-12, 2017.

20. Lavernia CJ and Sierra RJ: Core decompression in atraumatic osteonecrosis of the hip. J Arthroplasty 15: 171-178, 2000.

21. Lv H, Zhang L, Yang F, Zhao Z, Yao Q, Zhang L and Tang P: Comparison of microstructural and mechanical properties of trabeculae in femoral head from osteoporosis patients with and without cartilage lesions: A case-control study. BMC Musculoskelet Disord 16: 72, 2015.

22. Aldridge JM III, Berend KR, Gunneson EE and Urbaniak JR: Free vascularized fibular grafting for the treatment of postcollapse osteonecrosis of the femoral head. Surgical technique. J Bone Joint Surg Am 86: 87-101, 2004.

23. Wang BL, Sun W, Shi ZC, Zhang NF, Yue DB, Guo WS, Shi SH and Li ZR: Treatment of nontraumatic osteonecrosis of the femoral head using bone impaction grafting through a femoral neck window. Int Orthopaedics 34: 635-639, 2010.

24. Tsao AK, Roberson JR, Christie MJ, Dore DD, Heck DA, Robertson DD and Poggie RA: Biomechanical and clinical evaluations of a porous tantalum implant for the treatment of early-stage osteonecrosis. J Bone Joint Surg Am 87: 22-27, 2005.

25. Veillette CJ, Mehdian H, Schemitsch EH and McKee MD: Survivorship analysis and radiographic outcome following tantalum rod insertion for osteonecrosis of the femoral head. J Bone Joint Surg Am 88: 48-55, 2006.

26. Plakseychuk AY, Shah M, Varitimidis SE, Rubash HE and Sotereanos D: Classification of osteonecrosis of the femoral head. Reliability, reproducibility, and prognostic value. Clin Orthop Relat Res 386: 34-41, 2001.

27. Shuler MS, Rooks MD and Roberson JR: Porous tantalum implant in early osteonecrosis of the hip: Preliminary report on operative, survival, and outcomes results. J Arthroplasty 22 : 26-31, 2007.

28. Zhao D, Liu B, Wang B, Yang L, Xie H, Huang S, Zhang Y and Wei X: Autologous bone marrow mesenchymal stem cells associated with tantalum rod implantation and vascularized iliac grafting for the treatment of end-stage osteonecrosis of the femoral head. Biomed Res Int 2015: 240506, 2015.

29. Shi J, Chen J, Wu J, Chen F, Huang G, Wang Z, Zhao G, Wei Y and Wang S: Evaluation of the 3D finite element method using a tantalum rod for osteonecrosis of the femoral head. Med Sci Monit 20: 2556-2564, 2014.

30. Zhang Y, Li L, Shi ZJ, Wang J and Li ZH: Porous tantalum rod implant is an effective and safe choice for early-stage femoral head necrosis: A meta-analysis of clinical trials. Eur J Orthop Surg Traumatol 23: 211-217, 2013.

31. Wang C, Meng H, Wang Y, Zhao B, Zhao C, Sun W, Zhu Y, Han B, Yuan X, Liu R, et al: Analysis of early stage osteonecrosis of the human femoral head and the mechanism of femoral head collapse. Int J Biol Sci 14: 156-164, 2018
32. Liu Y, Zhao D, Wang W, Zhang Y, Wang B and Li Z: Efficacy of core decompression for treatment of canine femoral head osteonecrosis induced by arterial ischaemia and venous congestion. Hip Int 27: 406-411, 2017.

33. Castro FP Jr and Barrack RL: Core decompression and conservative treatment for avascular necrosis of the femoral head: A meta-analysis. Am J Orthop (Belle Mead NJ) 29: 187-194, 2000.

34. Arbab D and Konig DP: Atraumatic femoral head necrosis in adults. Dtsch Arztebl Int 113: 31-38, 2016.

35. Miao H, Ye D, Liang W and Yao Y: Effect of osteonecrosis intervention rod versus core decompression using multiple small drill holes on early stages of necrosis of the femoral head: A prospective study on a series of 60 patients with a minimum 1 year-follow-up. Open Orthop J 9: 179-184, 2015.

36. Liu Y, Su X, Zhou S, Wang L, Wang C and Liu S: A modified porous tantalum implant technique for osteonecrosis of the femoral head: Survivorship analysis and prognostic factors for radiographic progression and conversion to total hip arthroplasty. Int J Clin Exp Med 8: 1918-1930, 2015.

37. Mao Q, Wang W, Xu T, Zhang S, Xiao L, Chen D, Jin H and Tong P: Combination treatment of biomechanical support and targeted intra-arterial infusion of peripheral blood stem cells mobilized by granulocyte-colony stimulating factor for the osteonecrosis of the femoral head: A randomized controlled clinical trial. J Bone Miner Res 30: 647-656, 2015.

38. Huang $\mathrm{X}$, Jiang $\mathrm{H}$, Liu $\mathrm{D}$, Zhou $\mathrm{Z}$ and Wang L: Implantation of calcium phosphate cement/danshen drug delivery system for avascular necrosis of femoral head. Zhongguo Xiu Fu Chong Jian Wai Ke Za Zhi 22: 307-310, 2008 (In Chinese).

39. Jiang HJ, Huang XJ, Tan YC, Liu DZ and Wang L: Core decompression and implantation of calcium phosphate cement/danshen drug delivery system for treating ischemic necrosis of femoral head at Stages I, II and III of antigen reactive cell opsonization. Chin J Traumatol 12: 285-290, 2009.

40. Fan Y, Tang L and He W: Clinical study on the interventional treatment of non-traumatic femoral head mecrosis by integrated traditional Chinese and western medicine. Zhongguo Zhong Xi Yi Jie He Za Zhi 19: 605-606, 1999 (In Chinese).

41. Jiang $\mathrm{Y}$, Liu $\mathrm{C}$, Chen $\mathrm{W}$, Wang $\mathrm{H}$, Wang $\mathrm{C}$ and Lin $\mathrm{N}$ : Tetramethylpyrazine enhances vascularization and prevents osteonecrosis in steroid-treated rats. Biomed Res Int 2015: 315850, 2015.

42. Zheng SD and Wu HJ: Progress of studies on effects of ligustrazine on blood vessel endothelium protection. Zhongguo Zhong Xi Yi Jie He Za Zhi 31: 1004-1008, 2011 (In Chinese).

43. Liu Y, Yan L, Zhou S, Su X, Cao Y, Wang C and Liu S: Tantalum rod implantation for femoral head osteonecrosis: Survivorship analysis and determination of prognostic factors for total hip arthroplasty. Int Orthop 40: 1397-1407, 2016

44. Olsen M, Lewis PM, Morrison Z, McKee MD, Waddell JP and Schemitsch EH: Total hip arthroplasty following failure of core decompression and tantalum rod implantation. Bone Joint J 98: 1175-1179, 2016.

45. Cheng Q, Tang JL, Gu JJ, Guo KJ, Guo WS, Wang BL and Zhao FC: Total hip arthroplasty following failure of tantalum rod implantation for osteonecrosis of the femoral head with 5- to 10 year follow-up. BMC Musculoskelet Disord 19: 289, 2018.

(i) $(-)$ This work is licensed under a Creative Commons Attribution-NonCommercial-NoDerivatives 4.0 International (CC BY-NC-ND 4.0) License. 